Cinémas

Revue d'études cinématographiques

Journal of Film Studies

\title{
Les dispositifs de médiation dans le passage de l'architecture au cinéma
}

\section{Charles Perraton}

Volume 9, numéro 1, automne 1998

Les Dispositifs de médiation au cinéma

URI : https://id.erudit.org/iderudit/024771ar

DOI : https://doi.org/10.7202/024771ar

Aller au sommaire du numéro

Éditeur(s)

Cinémas

ISSN

1181-6945 (imprimé)

1705-6500 (numérique)

Découvrir la revue

Citer cet article

Perraton, C. (1998). Les dispositifs de médiation dans le passage de

l'architecture au cinéma. Cinémas, 9(1), 25-42. https://doi.org/10.7202/024771ar
Résumé de l'article

Le passage comme type architectural du XIXe siècle et le cinématographe comme mode spécifique de représentation du tournant du siècle dernier sont des dispositifs de médiation permettant aux individus d'inscrire leur rapport au monde dans des processus apparentés et complémentaires l'un de l'autre. Notre réflexion portera ici sur les rapports entre ces deux dispositifs qui, quoique hétérogènes à plusieurs égards, participent, chacun à leur façon, à la structuration des individus. 


\title{
Les dispositifs de médiation dans le passage de l'architecture au cinéma
}

\section{Charles Perraton}

\begin{abstract}
RÉSUMÉ
Le passage comme type architectural du XIX siècle et le cinématographe comme mode spécifique de représentation du tournant du siècle dernier sont des dispositifs de médiation permettant aux individus d'inscrire leur rapport au monde dans des processus apparentés et complémentaires l'un de l'autre. Notre réflexion portera ici sur les rapports entre ces deux dispositifs qui, quoique hétérogènes à plusieurs égards, participent, chacun à leur façon, à la structuration des individus.
\end{abstract}

\section{ABSTRACT}

The passage (a narrow street lined with shops) as an architectural type of the XIXth century and the cinematograph as a specific mode of representation which appeared at the end of the last century are both apparatuses of mediation which permit individuals to inscribe their relation to the world in interrelated, complementary processes. In this article we will consider the relationships between these two apparatuses, which, though different in many ways, both participate in their own fashion in the structuring of individuals.

Le passage comme type architectural du XIX siècle et le cinématographe comme mode spécifique de représentation de la fin du siècle dernier sont des dispositifs de médiation qui permettent aux individus d'inscrire leur rapport au monde dans des processus apparentés et complémentaires. Je considère donc ici que la 
médiation entre les individus et le monde se fait notamment à partir des rapports qu'ils entretiennent à travers une série de dispositifs plus ou moins abstraits ou concrets, matériels ou immatériels, qui organisent leurs pratiques. En même temps que les dispositifs de médiation naissent dans le contexte de rapports culturels particuliers, ils précèdent ces derniers dans la mesure où ils sont constitutifs du procès d'identification des individus (Crary, p. 18).

Ma réflexion portera ici sur les rapports entre ces deux dispositifs. Quoique hétérogènes à plusieurs égards, l'un d'action et l'autre de vision, ils participent, chacun à leur façon, à la strucuuration des individus. Ainsi, grâce au passage, ces derniers s'approprient et changent la ville à la faveur d'un essor du capitalisme, tout en entreprenant de se transformer eux-mêmes par leur nouveau mode d'habiter. Avec l'apparition des images projetées - des représentations de la lanterne magique jusqu'au cinématographe - le travail configurateur de l'imaginaire des individus se trouve suscité au profit d'expériences perceptives et de nouvelles perspectives éthique et esthétique de la communication.

Certes, le passage entretient des liens étroits avec d'autres archétypes architecturaux comme les galeries, les places publiques et les rues, et le cinématographe a des liens directs avec d'autres modes de représentation comme le panorama, le diorama, le phénakistiscope, le praxinoscope et le théatre optique, mais il m'intéressera surtout de connaître ici la parenté d'esprit entre ces dispositifs de médiation plutôt que de mesurer leur éventuelle filiation directe. Mon analyse portera surtout sur les conséquences symboliques (changement de l'ordre des représentations) et pragmatiques (les différentes manières qu'ont les dispositifs de positionner les corps) du passage et du cinématographe, l'un et l'autre considérés comme symptomatiques des changements culturels qui ont conduit à la production de l'individu moderne.

L'émergence de ces dispositifs pose le problème de leur usage et la question des rapports qu'ils génèrent entre les usagers, du fait niême qu'ils servent d'intermédiaire entre les usagers, et entre ceux-ci et le monde. Là se trouve selon moi la question 
proprement éthique. Comme Benjamin, je pense que dans le cas du passage, l'usage a tout de "[...] cet assujettissement avec lequel la propagande tant industrielle que politique doit pouvoir compter" (p. 51). Et nous verrons que la matérialité du dispositif est conçue pour en assurer le succès en contribuant à la production d'une nouvelle forme d'individu qui s'incarne plus ou moins dans la triple figure du citoyen (pour le nouvel État), du consommateur (pour la nouvelle économie) et du promeneur (pour la nouvelle idéologie) dans la cité.

Dans le cas du cinématographe, la nature même du dispositif appelle un élargissement de la notion d'usage, car si le visionnement (ou visionnage) des images en mouvement exige l'immobilisation d'un spectateur plongé dans l'obscurité, dès l'apparition des films à plusieurs plans, "[...] le film impose [...] au spectateur l'obligation de former dans son esprit l'image globale dont les élémerits lui sont successivement et non plus simultanément présentés » (Francastel, p. 184). C'est donc que le cinématographe se développe dans l'ambiguïté: en même temps qu'il contribue à l'émancipation des individus par l'éveil de l'imaginaire, il les introduit dans un mouvement de généralisation de l'image qui " [...] déréalise complètement le monde humain des confits et des désirs, sous couvert de l'illustrer" (Barthes, p. 182).

Mais parce qu'il favorise la production d'une nouvelle force d'expression chez les individus, on peut difficilement limiter le cinéma à la production et à l'actualisation de stratégies visant la gestion des comportements '; comme nous le verrons plus loin, il occupe une place de premier choix dans la configuration de mondes possibles, témoignant même d'une nouvelle manière d'expérimenter le monde.

\section{L'espace public au siècle des Lumières}

La naissance de l'espace public va de pair avec l'apparition d'un nouveau mode de communication sociale orientée vers l'élaboration de l'opinion publique. On en situe généralement l'émergence à la chute des pouvoirs centralisés représentés par la monarchie, alors qu'une nouvelle forme de sociabilité en vient à s'opposer aux hiérarchies et aux cloisonnements des pouvoirs. 
Inscrit dans une série de dispositifs de médiation, ce nouvel espace se développe dans de nouveaux lieux de production et de diffusion de l'information et du savoir. Pour Londres et Paris, c'est le cas des théâtres et des coffee-houses et, à un moindre degré, des jardins et des places publics.

L'espace public se constitue historiquement avec l'avènement de la communication impersonnelle. C'est-à-dire que les individus n'ont plus besoin de se référer à leurs particularités individuelles pour fonder la crédibilité de leurs propos et de leurs échanges. L'espace public devient dès lors le lieu d'expression d'une parole universelle acceptable pour tous, parce que débarrassée des contingences subjectives (Quéré, p. 45-82).

Tandis que les coffee-houses de la fin du XVII et du début du XVIII ${ }^{e}$ siècle sont reconnus comme des lieux consacrés au libre exercice de la parole, les jardins et les places apparaissent dès le milieu du XVIII siècle plutôt favorables à des contacts brefs entre les gens qui se croisent lors de promenades publiques qui finissent par changer "la structure des conversations" (Sennett, 1979, p. 76). Alors que l'opinion publique se déliait de toute attache sociale particulière, le souci des apparences devient peu à peu la nouvelle norme du rapport social et la communication sociale en vient à laisser la place à la communication des sentiments et des émotions des classes bourgeoises en quête d'identité. C'est dans ce contexte que l'architecture des passages s'élabore selon un régime de visibilité qui met en lumière de nouvelles figures de l'individu.

A ce que le siècle des Lumières avait laissé poindre comme horizon social des rapports communicationnels, le XIX ${ }^{e}$ siècle s'empresse de substituer une représentation courbe des rapports centrés sur «[...] la révélation directe des subjectivités [...]» et le «[...] déshabillage du moi» (Quéré, p. 59).

\section{Le passage comme type architectural du $\mathrm{XIX}^{\mathrm{e}}$ siècle}

Les grandes années des passages parisiens ont été celles qui précèdent l'arrivée de l'éclairage des rues au gaz. Tandis que les verrières recouvertes de vélum assurent un éclairage zénithal antiéblouissant, les lampes à l'huile les font briller la nuit dans le Paris de l'Empire "[...] comme des grottes habitées par des 
fées" (Benjamin, p. 581). Uniformisée, atténuée, diminuée, la lumière obtenue dans ces lieux crée en effet de bonnes conditions pour la mise en scène des objets et des individus qui s'y trouvent. Tamisé par les voiles, l'éclairage zénithal isole l'espace de son environnement naturel, l'homogénéise et lui donne une ambiance artificielle d' "Orient intérieur». L'espace devient propice aux fantasmagories des promeneurs.

La ville se donne à voir comme un "paysage urbain " pour la foule grouillante et l'intérieur du passage se meuble d'objets et d'éléments de décor desquels le promeneur espère tirer son inspiration (Benjamin, p. 877). Chacun, à l'écoute de son âme, interroge le miroir des objets exotiques qui s'y trouvent pour se faire unne «bonne image» de lui-même. La qualité esthétique et le caractère exotique des objets ont plus d'importance que leur utilité. Ils sont des petits luxes qui jouent un rôle prépondérant dans l'animation des passants, ces fantômes errants à la recherche d'une âme. Dès le début du XVIII siècle (1720), émerge un tel «[...] marché et une demande sociale d'objets liés au paraître social" (Nahoum-Grappe, p. 142). Le passage se fait de la valeur d'usage à la valeur d'échange. Le spectateur se fait ainsi luimême objet de mise en scène: "Tout un monde social [...] se donne en spectacle à lui-même dans l'ostentation de sa dimension esthétique» (p. 139).

Inaugurée au siècle précédent, l'institution de la promenade se cristallise au début du XIX siècle sous la forme achevée de la flânerie. Flâner ayant le sens de marcher çà et là, de se promener, gentiment, sans autre ambition que de passer le temps, en s'abandonnant à l'impression et au spectacle du moment. Or, l'action de flâner renvoie à celle de lanterner ${ }^{2}$, terme synonyme que l'on emploie au XVIII siècle pour décrire les promenades des aristocrates sous la lumière des lanternes. Activité qui ne pouvait d'ailleurs se réaliser sans présenter un certain nombre de risques (rapports malencontreux, vols et assauts, etc.) auxquels mit fin l'arrivée du passage comme espace public ouvert, mais contrôlé, qui ouvre donc sur l'extérieur tout en restant enfermé à l'intérieur. On pourrait avancer que la lanterne de l'époque aristocratique ("les aristocrates à la lanterne») laisse pour ainsi dire la place à l'éclairage zénithal des verrières de l'époque bourgeoise. 
Le nouveau dispositif de médiation assure ainsi le passage des contingences politiques et architecturales de l'espace public aux nécessités bourgeoises des espaces publics contrôlés. Il permet également de "dresser les corps" au profit d'une série de comportements adaptés aux nouvelles exigences économiques du capital. C'est que nous pouvons associer l'apparition des passages publics et l'invention du cinématographe au développement de dispositifs de médiation inscrits dans une perspective diagrammatique du pouvoir qui impose une certaine conduite aux individus. Foucault a en effet distingué le panoptique comme appareil formel et matériel qui permet de "voir sans être vu", du panoptisme comme principe général à l'œuvre dans les dispositifs de médiation qui visent à " [...] imposer une conduite quelconque à une multiplicité humaine quelconque» (1975, p. 41): "Chaque fois qu'on aura affaire à une multiplicité d'individus auxquels il faudra imposer une tâche ou une conduite, le schéma pourra être utilisé» (p. 207).

Le passage comme forme architecturale originale des grandes villes du XIX ${ }^{e}$ siècle inaugure un nouveau régime de rapports entre les citadins. C'est ce qui s'observe, par exemple, à la Galleria Vittorio Emanuele II de Milan, inaugurée en 1867, alors que la société industrielle italienne se trouve en plein essor. Ce passage contribue ${ }^{3}$ en effet à la production d'un nouveau "sujet", né de l'effort politique et architectural de structuration des individus; producteur tout à la fois d'un citoyen pour l'Italie unifiée, d'un consommateur pour les marchandises en vente dans la galerie marchande et d'un promeneur pour le nouvel espace public de propriété privée.

Le passage devient le symbole de la politique nationale. En se référant consciemment à Saint-Pierre de Rome, aux formes architecturales de l'Empire romain et à la croix du blason de la ville, qui allègue l'unité politique de l'Italie, le passage légitime le nouvel État en faisant appel à un large arrière-plan de traditions. Avec la Galleria Vittorio Emanuele, le type du passage entre dans la série des grands bâtiments représentatifs du XIX siècle, les théâtres, palais de justice, hôtels de ville, bourses, parlements, qui sont les manifestes de la société bourgeoise (Geist, p. 263). 


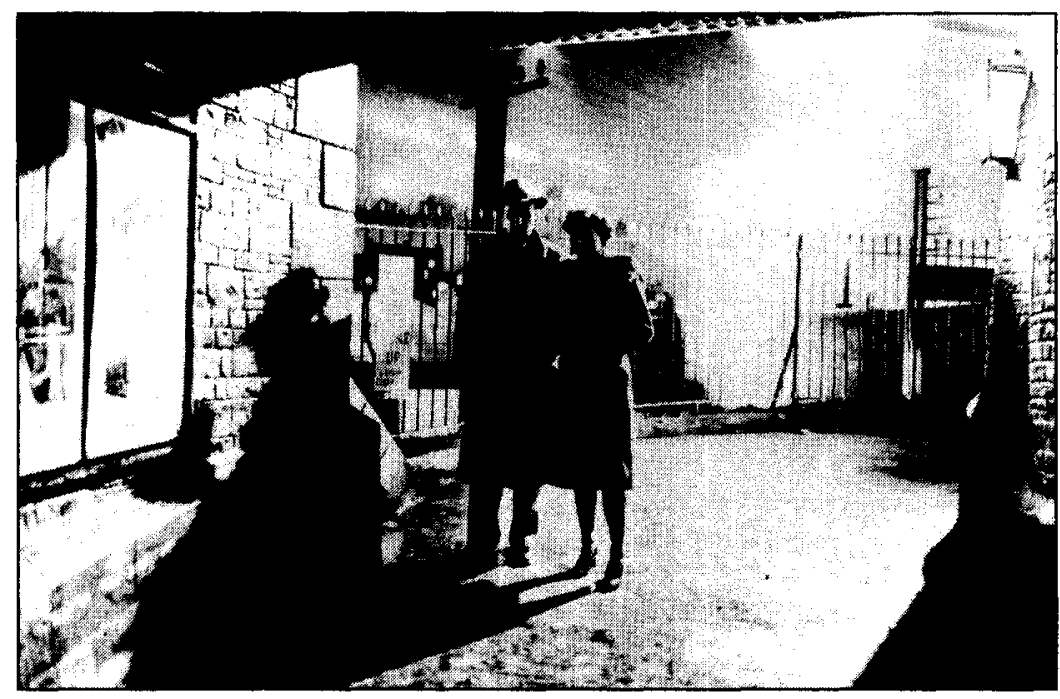

\section{Brève Rencontre de David Lean (1945)}

Le principe du passage est de l'ordre du panoptique, en ce sens que les passages publics se constituent et se construisent dans l'application du panoptisme qui vise à : "imposer une conduite quelconque à une multiplicité humaine quelconque». De plus, s'il est vrai que leur architecture se développe dans la reprise de certains éléments empruntés à la tradition architectonique des galeries et des musées, les passages publics n'en restent pas moins une traduction originale et (apparemment) inoffensive du panoptisme dont les premières applications se trouvaient plutôt du côté pénitencier.

Symbolique, commerciale et pragmatique, la rationalité architecturale et politique du passage conjugue donc ouverture et mise en lumière de manière à horizontaliser et à intensifier les nouveaux rapports entre les individus. À la raison architecturale et politique de la fin XVIII et du début XIX ${ }^{e}$ siècle, le passage apparaît non seulement comme un moyen de mettre à profit (au sens propre de la spéculation foncière) les espaces interstitiels de la cité, mais aussi une façon de redonner à ses habitants ce que la ville leur avait enlevé pour assurer son développement. Lélaboration des passages s'est ainsi faite dans le but exprès de "réinventer " la ville en fonction de nouveaux impératifs commerciaux, 
symboliques et pragmatiques. Or ce qui était concédé d'une main était repris de l'autre, puisque ce nouvel espace public sur lequel ouvrait le passage était de propriété et de gestion privées. C'est d'abord en ce sens que je peux dire que "le passage du XIX siècle marque le triomphe de la vie privée sur la sphère publique».

\section{Le passage des Panoramas}

L'un des passages les plus célèbres, et parmi les premiers à avoir vu le jour (1800), est sans contredit le passage des Panoramas. Une passerelle y réunit deux panoramas énormes qui ont plus à partager avec ce dernier que le simple fait d'appartenir au même lieu. Leur inclusion dans le passage en fait des dispositifs emboîtés de médiation. Loin d'être fortuit, leur emboîtement renforce leurs liens structuraux.

Au tournant du XIX ${ }^{e}$ siècle apparaît en effet un nouveau genre $^{4}$ de peinture, le panorama, fait d'un " [...] tableau peint sur une toile de très grande dimension (jusqu'à 10 ou 15 mètres de haut sur 100 à 120 mètres de long) développé circulairement sur le mur intérieur d'une rotonde éclairée par le haut et spécialement construite pour la recevoir" (Laclotte, p. 1360). Ce nouveau genre de peinture tend, pour l'essentiel, à « [...] donner l'illusion de la réalité grâce aux diverses ressources du trompel'œil" (p. 1360). Daguerre (1787-1851), qui s'associa plus tard, en 1839, à Niepce (1765-1833), l'inventeur de la photo, pour la rendre publique en la fixant sur daguerréotype, excellait dans l'exécution de ces peintures et inaugura même une série de moyens qui révolutionnèrent en peu de temps l'art des effets de scène. En effet Daguerre développa, selon Benjamin 5, l'idée même du cinéma appliquée à des tableaux, l'idée "d'un cinéma antérieur à la photographie ".

Le diorama de Daguerre présente, en 1822, une innovation technique intéressante: les spectateurs, assis dans l'obscurité sur un plateau mobile, sont mis successivement en présence de tableaux animés par un jeu de lumières sur des plans transparents superposés; ainsi obtient-on, avec l'illusion du relief, des effets de neige et de brouillard tout à fait surprenants (Bertier de Sauvigny, p. 374). 
Le panorama propose donc au spectateur un nouveau type d'expérience. Il lui permet de parcourir de nouveaux horizons tout en restant immobile. C'est là une préoccupation d'époque, puisqu'on expérimente déjà avec les voitures (cabriolets et omnibus), ou on expérimentera sous peu avec le train, cet «[...] impératif du détachement qui oblige à payer une abstraite maîtrise oculaire de l'espace en quittant tout lien propre, en perdant pied» (Certeau, p. 201).

Entre le passage et le panorama, il faut toutefois remarquer une différence dans la place qui est ménagée à l'usager. En effet, l'usager du passage se trouve au cour d'un spectacle auquel il assiste, et dans lequel il joue son propre rôle, alors que l'usager du panorama assiste à un spectacle dans lequel il ne joue pas, mais où lui est offerte l'illusion de se trouver au milieu de la scène. Illusion d'autant plus grande que «[...] les vues que doivent reproduire les panoramas furent d'abord levées à la chambre noire sur pivot tournant, permettant de dessiner tout l'horizon " (Sadoul, p. 27).

On comprend que s'il s'en éloigne, le passage appartient encore à l'univers culturel du XVIII ${ }^{e}$ siècle, puisqu'il laisse un rôle actif à l'usager qu'il transforme par ailleurs en spectateur. Comme dispositif de vision, il met paradoxalement la foule et les objets en lumière. Mais en isolant le lieu du reste de la ville, il contribue à la réinvention de cette dernière par la production de nouveaux espaces publics. Et en extrayant des individus de nouvelles forces politique, économique et idéologique, il contribue à la production de l'individu moderne. Pour sa part, le panorama précise le rôle du spectateur en le plongeant dans l'obscurité à la faveur d'une mise en lumière des tableaux qu'il lui offre en spectacle ${ }^{6}$, travaillant ainsi à la production d'un public pour les nouveaux espaces.

\section{Du passage au cinématographe}

Par ailleurs, les travaux du Belge Joseph Plateau (1801-1883) sur la résistance rétinienne le conduisent, en 1832, à inventer le phénakistiscope, appareil qu'il met au point pour faire la synthèse du mouvement. Ce dispositif de vision a la forme d'un disque fenêtré avec des intervalles plus ou moins courts et 
suffisamment rapprochés de manière à faire correspondre à chaque fenêtre une image fixe qui s'anime lorsque le dispositif est mis en mouvement. Cette invention de Plateau permet de "[...] transformer un mouvement rapide en un mouvement aussi lent qu'on le désire" (Sadoul, p. 14). Or, le passage et le panorama ont beaucoup en commun avec ce principe de décomposition du mouvement.

Dans le cas du panorama, le dispositif segmente le paysage au profit d'une narration et de sa mise en scène ${ }^{7}$, et il reconstitue le mouvement au rythme fixé par la plate-forme sur laquelle prend place le spectateur immobile. Pour sa part, le passage contribue comme dispositif à fixer les rythmes de déplacement ${ }^{8}$. Chaque vitrine se présente en effet comme un moment de la promenade et met en scène les objets en les cadrant et en les éclairant. Par son regard porté sur les objets mis en scène dans les vitrines et par son parcours intermittent, le flâneur anime le dispositif comme s'il était déjà l'opérateur et le personnage d'un phénakistiscope. Dans les deux cas, les dispositifs de médiation font passer de l'action à la vision comme mode pragmatique réduit et singulier d'action. Ainsi pourrions-nous dire que les panoramas assurent le passage de l'action à la vision, le passage de l'architecture au cinéma.

L'Anglais Horner donne une forme cylindrique au phénakistiscope de Plateau en 1834 et il le baptise zootrope. Avec son invention, il substitue au disque un cylindre fenêtré sur la face intérieure duquel il installe une bande d'images qui reprend le principe circulaire de la toile du panorama et qui préfigure le film. Sur cette bande, il fait apparaître des figures représentant les différents moments du geste d'un personnage qui s'anime au moindre mouvement de l'appareil.

Également intéressé par les travaux de Plateau et soucieux de trouver un moyen de projeter de telles images en mouvement, le Français Émile Reynaud perfectionne le zootrope, en 1877, en plaçant au centre de l'appareil des miroirs (dont le nombre correspond à la quantité d'images qui lui font face) qui réfléchissent (moyen rudimentaire de projection) les images disposées sur le tambour. Il nomme son appareil praxinoscope (comme le fait remarquer Laurent Mannoni, le terme praxinoscope vient du grec praxis, «action» et scopeo, "je regarde»; de sorte que 
l'action échappe dorénavant à celui dont la fonction du regard devient déterminante), auquel il combine, peu de temps après, les principes de la lanterne magique pour donner au musée Grévin du passage Jouffroy, le 28 octobre 1892, le premier spectacle de théâtre optique. Il faut préciser que dans le théâtre optique, le miroir devient un élément déterminant du dispositif puisque, grâce à lui, les personnages de la bande peuvent pour ainsi dire sortir du praxinoscope et produire des effets de profondeur et de réalisme tout à fait saisissants. Le miroir ouvre la porte à l'imaginaire et l'écran devient magique. Les découvertes en chronophotographie de Muybridge (1878) et de Marey (1882) ajouteront l'essentiel de ce qui manque à Louis Lumière pour achever le travail nécessaire à l'invention du cinématographe (1895), comme ultime dispositif de vision qui fixe la médiation de l'espace autour de la représentation.

À travers l'évolution de ces différents dispositifs, nous voyons apparaître graduellement le principe du cinématographe comme dispositif de synthèse et de reproduction du mouvement, comme "machine à refaire la vie» (Sadoul, p. 275).

\section{Du cinématographe au cinéma}

L'arrivée du cinématographe inaugure d'autres modalités de réception et soulève de nouvelles questions quant au sens et à l'usage des dispositifs de médiation. Il contribue ainsi à l'émergence du spectateur-interprète comme nouvelle forme de l'individu pour qui se profilent à l'horizon, au-delà des dimensions instrumentale et esthétique, les dimensions éthique et sociale du langage. Entre le train et son image dans L'Arrivée du train en gare de La Ciotat (Louis Lumière, 1895), il y a en effet le spectateur qui en saisit le sens et la portée au moment de son interprétation " [...] ce qui attira les premières foules ce ne fut pas une sortie d'usine, un train entrant en gare (il aurait suffi d'aller en gare ou à l'usine) mais une image du train, une image de sortie d'usine» (Morin, 1965, p. 16).

Le film fait sortir le spectateur de son autonomie pour le mêler au flux des regards "[...] que draine le sortilège de l'écran» (Agel, p. 48). En ce sens, il « [...] fait appel à une collaboration de l'intelligence du public" (p. 52); l'espace et le 
temps qu'il propose n'existent que dans la mesure où ils sont construits à la rencontre du film et de son spectateur. Le charme des images se manifeste dès les premiers films. Il tient à leur capacité de susciter le travail configurateur de l'imaginaire, aussi bien celui des opérateurs Lumière qui réalisèrent les premières «vues» que celui des spectateurs d'hier et d'aujourd'hui.

C'est cette voie des configurations imaginaires qui permet de réinventer le monde plutôt que de nous y soumettre. Le film est en ce sens une proposition de monde qui naît des images et du passage de l'une à l'autre; passage qui ne peut être assuré sans le concours du spectateur. L'image qu'il donne du monde se trouve faite «[...] d'innombrables "points de vue" [qui] contribuent à faire naître en lui une vision synthétique de l'espace" (Francastel, p. 185). Depuis Lang (Metropolis, 1927) et Vertov (L'Homme à la caméra, 1929) au moins, le cinéma s'énonce aussi sous une forme réflexive qui permet au spectateur de prendre du recul qui n'a rien de la distance perspectiviste impliquée par le dispositif de mise en forme cinématographique de l'espace - sur ses habitudes de réception en lui donnant l'occasion d'inférer le sens des images non seulement en "[...] fonction du contexte filmique créé par le montage [...]" mais aussi en fonction de son propre "[...] contexte mental [de] spectateur" (Martin, p. 29) et du contexte institutionnel de réception (Odin, 1983).

Il s’agit de réaliser une série d'images composées de relle sorte qu'elle provoque un mouvement affectif qui éveille à son tour une série d'idées. De l'image au sentiment, du sentiment à la thèse [...] je pense que seul le cinéma est capable de faire cetre grande synthèse, de rendre à l'élément intellectuel ses sources vitales, concrètes et émotionnelles (Eisenstein, cité dans Barbèche, p. 344).

Plus tard, dans Rear Window (1954), Hitchcock met en œuvre un dispositif qui aide à comprendre la portée réflexive du film par la distinction qu'il fait entre deux types d'espace: l'un qui donne à voir (le lieu d'où l'on voit) et l'autre qui est donné à voir (le lieu que l'on voit). À la chambre du héros, Jeffries (James Stewart), s'ajoute l'espace du mur d'en face qui agit comme écran sur lequel défilent les images qu'il observe avec ses 


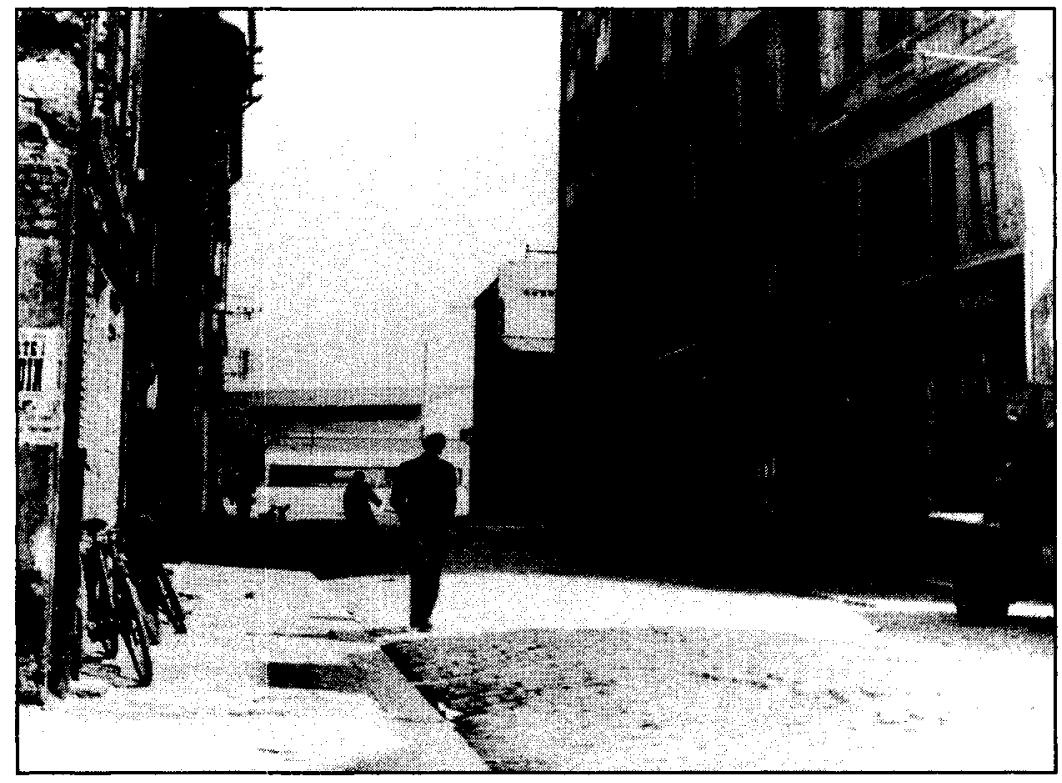

\section{Un homme marche dans la ville de Marcello Pagliero (1950)}

jumelles et son téléobjectif. La cour intérieure devient la destinée de tous les regards, l'occasion de toutes les inférences.

Or, grâce à ce dispositif, Hitchcock nous fait passer d'un cinéma narratif de "la" représentation (comme calque du réel) à un cinéma réflexif "des" représentations (comme perspectives), mettant de l'avant l'idée qu'il y a autant de représentations possibles qu'il s'y trouve de points de vue dans et sur le film. On peut penser qu'il insiste sur cette idée du fait que le contexte de réception des films s'est lourdement institutionnalisé depuis notamment l'apparition des genres au cinéma, à un point où il est devenu difficile de voir sans chercher à comprendre, de comprendre sans chercher l'histoire qui organise les personnages et les événements entre eux. Pour Hitchcock, ce qui est vu l'est par l'intermédiaire du personnage: Jeffries voit et retrouve sur l'écran d'en face ce qu'il y a mis, de la même façon que le spectateur retrouve dans le film ce que le réalisateur y a mis pour l'interpréter.

Par là, Hitchcock pose, expose et fait en sorte qu'explosent les règles du cinéma classique - au sens où la colonne grecque est 
classique, puisqu'elle ne sert pas seulement de soutien, mais elle exprime aussi sa fonction, au sens où, par sa forme, elle tient un discours sur elle-même. Et même s'il lui a fallu nous le rappeler, il apparaît maintenant clair que le film n'est pas la seule affaire du réalisateur, mais aussi celle du spectateur et que le sens de l'œuvre devient le produit de leurs efforts conjugués. L'auteur y est aussi interprète que l'interprète y est auteur.

\section{La portée éthique de l'interprétation}

Me situant sous l'angle sémio-pragmatique", le produit d'un dispositif de médiation reste pour moi - qu'il s'agisse d'un dispositif d'action (l'espace architectural) ou d'un dispositif de vision (le film) - fait d'énoncés que nous devons comprendre et interpréter. Il doit donc «[...] être considéré comme un objet que l'interprétation construit [...] dans le but, circulaire, de se valider elle-même sur la base de cela même qu'elle construit " (Eco, p. 23). C'est que les espaces et les images ne prennent leurs significations idéologique, éthique et sociale que dans l'attention spectatorielle, à la recherche de l'intentionnalité de l'auteur à partir de ce qu’en révèle le texte.

Dans ce travail d'interprétation ${ }^{10}$, l'espace ou le film se poursuivent ou se refont par l'enchaînement d'un nouveau discours au discours du texte (Ricœur, p. 152). Et c'est, je crois, dans la communauté des sujets que se médiatise la dynamique entre exprimer et interpréter, dire et enchaîner. Cette médiation par le discours est le lieu de l'«Institution " qui réunit constitutivement les sujets et se trouve constituée par eux.

Les communautés interprétatives peuvent alors se présenter comme l'horizon d'une nouvelle sociabilité qui éloigne du politique (les formes sociales d'organisation) au profit des individus et du langage. Pour reprendre Tzvetan Todorov, je dirais qu'elles permettent de $[\ldots]$ retrouver le sens du social sans perdre la qualité de l'individuel» (p. 253). Ainsi, pour éviter les obstacles communicationnels engendrés par le procès de personnalisation qu'illustre bien le passage comme type architectural du XIX siècle, je pense comme Louis Quéré qu'il y a lieu de «[...] maintenir un clivage fondateur entre les sujets sociaux et quelque chose qui, parce qu'il reste distinct d'eux, préserve leurs 
différences et leurs intimités tout en leur permettant de se lier entre eux" (p. 62).

Depuis sa naissance, à la fin du siècle dernier, le cinéma y contribue sans doute mieux que tout autre dispositif de médiation. Dans tous les cas, l'enjeu de l'interprétation réside dans le sens à donner au texte, ce qui se fait en respect ou au mépris d'autrui qui est aussi bien ce dont parle le texte que ce pour quoi (pour qui) il parle. Les liens qu'assurent les communautés interprétatives ne limitent en rien la portée éthique de l'interprétation, ils peuvent même au contraire faire en sorte que soit satisfaite l'exigence d'expression des individus, aussi bien dans l'acte de création que dans celui de l'interprétation des œuvres ${ }^{12}$. L'un et l'autre, mais selon des modalités différentes, engagent la singularité de l'individu qui, par son geste ${ }^{13}$, réfléchit ses conditions d'existence au double sens du miroir qui permet à la fois de les saisir (reflet) et de les dépasser (passage).

On a vu que l'émergence des dispositifs de médiation pose le problème de leur usage et la question des rapports qu'ils génèrent, du fait même qu'ils servent d'intermédiaire entre les individus et le monde. La nature de ces dispositifs qui entourent la naissance du cinéma et les usages auxquels ils ont donné lieu jusqu'à maintenant ont largement contribué à la production d'une nouvelle manière de voir et d'habiter le monde.

Si la première tâche du cinéma naissant aura été d'ordre architectural en organisant " [...] le volume virtuel à partir de l'orthogonalité définie par les lois de l'optique et le code de la perspective» (Gardies, p. 14), celle de l'architecture du tournant du XIX siècle aura été d'ordre cinématographique en produisant un nouveau mode cinématique d'habiter la ville (sans autre implication que visuelle), qui neutralise les lieux par le désinvestissement des significations. Pour Richard Sennett en effet, la ville devient à partir du XIX siècle une suite d' " [...] étalages séquentiels et linéaires de différences [...]" à laquelle l'œil du passant ne réagit plus qu'avec indifférence (1992, p. 163-164), ce qui s'évalue à la manière dont ce dernier voit la puissance au travail dans l'espace. Sennett voit la solution à cette difficulté dans la réhabilitation du pouvoir d'interprétation de l'œil qui, plutôt que de s'en remettre aux représentations du pouvoir 
(celles qui permettent de penser que chacun est maître de luimême), doit tenir sa conscience en éveil en expérimentant visuellement la ville d'une autre manière, et notamment par l'expérience non linéaire de la différence.

Or, s'il est vrai, pour reprendre la thèse de Sandro Bernardi, que grâce au cinéma, ce que nous avons vu et expérimenté, c'est finalement "l'acte de regarder" (p. 23), il faudra donc partir du cinéma (partir de là, mais en sortir ensuite) pour réinventer la ville.

\section{Université du Québec à Montréal}

\section{NOTES}

1 Il faut se demander si le cinéma classique de la grande période des genres n’a pas reconduit sous une forme quelque peu "anesthésiante" ce projet d'imposer une conduite aux individus par l'entreprise de gestion des corps (le spectateur assis à sa place) et des âmes (l'imaginaire subsumé sous le régime narratif d'agencement des images).

2 Lanterner signifie en 1773: "[...] perdre son temps en s'amusant à des riens, ou par irrésolution; faire lanterner: faire attendre” (Le Robert).

3 Sans doute mieux que tout autre, mais de manière comparable à la galerie d'Orléans du Palais-Royal, construite entre 1828 et 1830.

4 Pour nous, il s'agit véritablement d'un nouveau type de dispositif de médiation, puisqu'il se développe sur de nouveaux supports et en de nouveaux lieux.

5 «En cherchant à produire dans la représentation de la nature des modifications très réalistes de genre, les panoramas [annonçaient], par-delà la photographie, le film et même le film sonore" (Walter Benjamin, Paris, capitale du XIX siècle. Le livre des passages, Paris: Cerf, 1989, p. 37).

6 Dans les faits, comme l'indique Foucault, «[...] nous ne sommes ni sur les gradins ni sur la scène, mais dans la machine panoptique, investis par ses effets de pouvoirs que nous reconduisons nous-mêmes puisque nous en sommes un rouage" ( $S_{t u \text { - }}$ veiller et punir. Naissance de la prison, Paris: Gallimard, 1975, p. 279).

7 Walter Benjamin précise: «[...] on s'évertuait infatigablement à transformer les panoramas, par des artifices techniques, en théâtres d'une parfaite imitation de la nature. On s'efforçait de reproduire les changements de la lumière du jour dans le paysage, le lever de la lune, le bruissement des cascades" (Walter Benjamin, Paris, capitale du XIX siècle. Le livre des passages, Paris: Cerf, 1989, p. 37).

8 Au sujet de cette vitesse, Benjamin raconte qu'en 1839, "[...] il était élégant d'emmener une tortue quand on allait se promener" (Walter Benjamin, Paris, capitale du XIX siècle. Le livre des passages, Paris: Cerf, 1989, p. 441).

9 Jusqu'ici, c'était plutôt sous l'angle anthropologique que j'abordais les dispositifs de médiation, les considérant alors comme des visibilités changeantes selon les contextes culturels. C'est donc que je reprends ici la distinction foucaldienne entre les mots et les choses: "[le langage] forme la tache aveugle à partir de quoi les choses autour de nous se disposent comme nous les voyons aujourd'hui " (L'Archéologie du savoir, Paris: Gallimard, 1969, p. 262-263). 
10 Comprenons que l'interprétation ne se manifeste pas toujours sous sa forme maximisée. L'imaginaire est en effet plus ou moins évoqué et convoqué selon les œuvres, le contexte et les individus. N'agissons-nous pas aussi beaucoup par habitude? C'est même indispensable, sans quoi nous ne bougerions plus sous le poids de nos responsabilités interprétatives.

11 L' Institution" est faite des règles, conventions et pratiques produites par (et productrices) des individus membres des diverses communautés interprétatives. Elle est le lieu d'actualisation, de négociation et de production de ces règles et du sens des pratiques communicationnelles qui lient les membres de la communauté.

12 J'emploie "œuvre" au sens que lui donne Étienne Souriau, c'est-à-dire de "responsabilité à l'égard du monde inachevé" (La Couronne d'herbes, Paris: Union générale d'éditions, 1975); ce qui vaut pour les textes qui restent toujours à (re)faire. Commentant Souriau, Mikel Dufrenne note par ailleurs que: "[...] l'œuvre réfere doublement à l'expérience esthétique: à celle du créateur pour être accomplie, à celle du récepteur pour être appréciée" ("La couronne d'herbes", L'Art instaurateur, Paris: Union générale d'éditions, 1980 , p. 99).

13 Son geste correspond à " [...] cet effort par lequel je deviens ce que je suis ou je puis le devenir, hors duquel il n'est point d'existence au sens strict [...]" (Michel Morin, L'Amérique du Nord et la culture, Montréal, Hurtubise-HMH, 1982, p. 57).

\section{OUVRAGES CITÉS}

Agel, Henri. «Activité ou passivité du spectateur», dans Étienne Souriau (direction), L'Univers filmique. Paris: Flammarion (1953), p. 47-58.

Barthes, Roland. La Chambre claire. Paris: Gallimard, 1980.

Barbèche, Maurice et Robert Brasillach, Histoire du cinéma, tome 1: Le cinéma muet. Paris: Sept couleurs, 1964.

Bernadi, S. Le Regard esthétique ou la visibilité selon Kubrick. Paris: Presses universitaires de Vincennes, 1994.

Benjamin, Walter. Walter Benjamin, Paris, capitale du XIX siècle. Le livre des passages. Paris: Cerf, 1989.

Bertier de Sauvigny, G. de. Nouvelles histoires de Paris. La Restauration 1815-1830. Paris: Hachette, 1977.

Certeau, Michel de. LInvention du quotidien, tome I. Arts de faire. Paris: Minuit, 1980. Crary, Jonathan. Tecinniques of the Observer. Cambridge: MIT Press, 1990.

Dufrenne, Mikel. "La Couronne d'herbes", L'Art instaurateur. Paris: Union générale d'éditions (1980), p. 96-117.

Eco, Umberto. «Notes sur la sémiotique de la réception». Actes sémiotiques, vol. IX, $\mathrm{n}^{\circ} 81$ (1987), $27 \mathrm{p}$.

Eisenstein, S.M. Le Film, sa forme, son sens. Paris: Bourgois, 1976.

Foucault, Michel. LAArchéologie du savoir. Paris: Gallimard, 1969.

Foucault, Michel. Surveiller et punir. Naissance de la prison. Paris: Gallimard, 1975.

Francastel, Pierre. L'Image, la vision et l'imagination. Paris: Gonthier, 1983.

Gardies, André. "La cité Lumière». CinémAction, n"75 (1995), p. 12-16.

Geist, J.-F. Le Passage. Un type architectural du XIX siècle. Bruxelles: Pierre Mardaga, 1989.

Laclotte, Michel (direction). "Panorama", Petit Larousse de la peinture. Paris: Larousse (1979), p. 1360. 
Mannoni, Laurent. Le Grand Art de la lumière et de l'ombre. Archéologie du cinéma. Paris: Nathan, 1995.

Martin, Marcel. Le Langage cinématographique. Paris : Cerf, 1985.

Morin, Edgar. Le Cinéma ou l'homme imaginaire. Paris: Gonthier, 1965.

Morin, Michel. L'Amérique du Nord et la culture. Montréal : Hurtubise-HMH, 1982

Nahoum-Grappe, V. "Conflit de parure et vouloir paraître: briller à Paris aux XVIII" siècle", Communications, n" 46 (1987), p. 135-156.

Odin, Roger. "Pour une sémio-pragmatique du cinéma", Iris, vol. 1, n" 1 (1983), p. 67-83.

Quéré, Louis. Des miroirs équivoques. Aux origines de la communication moderne, Paris: Aubier-Montaigne, 1982.

Ricœur, Paul. "Qu'est-ce qu'un texte?", Du texte à l'action. Paris: Seuil (1986), p. 137-159.

Sadoul, Georges. Histoire générale du cinéma, tome I. Linvention du cinéma, 18321897. Paris: Denoël, 1948.

Sennett, Richard. Les Tyrannies de l'intimité. Paris: Seuil, 1979.

Sennett, Richard. La Ville à vue d'ail. Paris: Plon, 1992.

Souriau, Étienne. La Couronne d'herbes. Paris: Union générale d'éditions, 1975.

Todorov, 'T. La Conquête de l'Amérique. La question de l'autre. Paris: Seuil, 1982. 